\title{
Oxygen targeting in preterm infants using the Masimo SET Radical pulse oximeter
}

\author{
Ewen D Johnston, ${ }^{1}$ Breidge Boyle, ${ }^{2}$ Ed Juszczak, ${ }^{2}$ Andy King, ${ }^{2}$ Peter Brocklehurst, ${ }^{2}$ \\ Ben J Stenson ${ }^{1}$
}

'Simpson Centre for Reproductive Health, Royal Infirmary of Edinburgh, Edinburgh, UK

${ }^{2}$ National Perinatal Epidemiology Unit, University of Oxford, Oxford, UK

\section{Correspondence to} Dr B J Stenson, Simpson Centre for Reproductive Health, Royal Infirmary of Edinburgh, Edinburgh EH16 4SA, UK;

ben.stenson@luht.scot.nhs.uk

Accepted 26 January 2011 Published Online First 6 March 2011

\begin{abstract}
Background A pretrial clinical improvement project for the BOOST-II UK trial of oxygen saturation targeting revealed an artefact affecting saturation profiles obtained from the Masimo Set Radical pulse oximeter. Methods Saturation was recorded every 10 s for up to 2 weeks in 176 oxygen dependent preterm infants in 35 UK and Irish neonatal units between August 2006 and April 2009 using Masimo SET Radical pulse oximeters. Frequency distributions of $\%$ time at each saturation were plotted. An artefact affecting the saturation distribution was found to be attributable to the oximeter's internal calibration algorithm. Revised software was installed and saturation distributions obtained were compared with four other current oximeters in paired studies.
\end{abstract}

Results There was a reduction in saturation values of $87-90 \%$. Values above $87 \%$ were elevated by up to $2 \%$, giving a relative excess of higher values. The software revision eliminated this, improving the distribution of saturation values. In paired comparisons with four current commercially available oximeters, Masimo oximeters with the revised software returned similar saturation distributions.

Conclusions A characteristic of the software algorithm reduces the frequency of saturations of 87-90\% and increases the frequency of higher values returned by the Masimo SET Radical pulse oximeter. This effect, which remains within the recommended standards for accuracy, is removed by installing revised software (board firmware V4.8 or higher). Because this observation is likely to influence oxygen targeting, it should be considered in the analysis of the oxygen trial results to maximise their generalisability.

\section{INTRODUCTION}

Preterm infants often require supplemental oxygen for prolonged periods. There is considerable interest in the effect of oxygen therapy on morbidity and mortality in these infants. ${ }^{1-6}$ For more than 60 years there has been insufficient evidence to guide treatment and this is now the subject of an international group of randomised trials. The aim of these trials is to compare the outcomes of extremely preterm infants who have their pulse oximeter saturation $\left(\mathrm{SpO}_{2}\right)$ maintained in either the range $85-89 \%$ or $91-95 \%$ until they reach 36 weeks corrected gestational age. The trials are using Masimo SET Radical pulse oximeters (Masimo, Irvine, California, USA). These oximeters were chosen because they were in widespread use when the trials were planned and are popular because of their ability to measure during patient motion and in circumstances of low perfusion.

\section{What is already known on this topic}

- Different oxygen targeting practices may influence the short and long term outcomes of preterm infants.

- International trials are being conducted with the aim of ending 60 years of uncertainty about oxygen therapy for preterm infants.

\section{What this study adds}

- A software characteristic causes the Masimo SET Radical oximeter to return fewer saturation values between $87 \%$ and $90 \%$ and an excess of higher values.

- This may affect oxygen targeting and can be removed by installing revised software (board firmware V4.8 or higher).

- The international trials will produce a pooled result that is widely generalisable.

The trials were made possible by the assistance of Masimo Corporation who provided modified oximeters to enable a masked comparison between the trial groups.

Prior to commencing the BOOST-II UK trial (https://www.npeu.ox.ac.uk/boost), participating units provided routine care to convalescent oxygen dependent preterm infants using standard unmodified Masimo SET Radical oximeters and participated in a pretrial audit to evaluate and support their success in saturation targeting. During this audit, $\mathrm{SpO}_{2}$ data were recorded in the oximeters and were downloaded for analysis and feedback to the clinical teams. In the course of this analysis, it was noted that the frequency histograms of the $\mathrm{SpO}_{2}$ readings had an unexpected appearance. The purpose of this report is to describe the observation and discuss its relevance to clinical practice and the oxygen saturation targeting trials.

\section{METHODS}

$\mathrm{SpO}_{2}$ data were gathered from 176 oxygen dependent preterm infants in $35 \mathrm{UK}$ and Irish neonatal units between August 2006 and April 2009. Monitoring was performed for normal clinical indications using standard, unmodified Masimo SET Radical oximeters. Care was guided by these oximeters for a period of up to 2 weeks. During this time, the $\mathrm{SpO}_{2}$ data were recorded by the 
oximeter every $10 \mathrm{~s}$. The infants were being given standard care by regular neonatal unit staff using standard equipment with no additional interventions or observations. As this was an audit of normal care for quality improvement purposes, parental consent was not obtained and ethics committee approval was not required. At the end of the period of care, the $\mathrm{SpO}_{2}$ data were downloaded and fed back to the clinical team so that the staff could evaluate their success with saturation targeting. The feedback was provided as bar charts describing the time spent in different saturation ranges.

Alternative ways of describing the data were explored and the $\mathrm{SpO}_{2}$ data were later plotted in the form of frequency histograms of the percentage of $\mathrm{SpO}_{2}$ readings at each saturation. When the saturation distributions were reviewed and appeared to have an artefact modified appearance, work was undertaken by Masimo Corporation to explain the findings. The explanation lay in the oximeter software algorithm. A revised software algorithm was supplied by Masimo Corporation and installed into the oximeters. Paired studies comparing the saturation distributions returned by Masimo oximeters running the new software algorithm with commercially available oximeters from four other manufacturers were conducted on a series of preterm infants cared for in the neonatal unit of the Royal Infirmary of Edinburgh, UK. Written parental consent was obtained for each of the paired comparisons and the protocol for the paired comparisons was reviewed by the Lothian Research Ethics Committee who determined that a full ethics application and review was not required because the study was an equipment evaluation with no modification to patient care other than the attachment of an additional oximeter. Probes from both oximeters were sited postductally for these comparisons.

\section{RESULTS}

Pretrial audit data were obtained from 176 preterm infants; all were receiving supplemental oxygen at the time of study. Detailed clinical information was not gathered as the audit was not intended as a research study. Figure 1A shows the frequency histogram of saturation readings from one infant. The distribution has an artefactual appearance, with a large dip in the histogram due to a lower frequency of $\mathrm{SpO}_{2}$ readings in the range $87-90 \%$ than would be expected from the remainder of the distribution. It looked as if there were missing values. This appearance was identifiable in almost all histograms from individual infants. The area of apparently missing data always fell in the range $87-90 \%$. The size of the area of missing values depended on the distribution of the remaining values. Where an $\mathrm{SpO}_{2}$ of 87-90\% was in the middle of the patient's distribution, the dip appeared large. Where the centre of the distribution was above a saturation of $90 \%$ so that values of $87-90 \%$ were in the tail, the dip appeared smaller. In a few cases with very high $\mathrm{SpO}_{2}$ values, the dip was not apparent as there were too few values in the range $87-90 \%$. This is shown in figure $1 \mathrm{~B}$ where histograms from five infants have been plotted, and in figure $1 \mathrm{C}$ where pooled data from all 176 audits have been combined, showing the overall effect on the saturation distribution of oxygen dependent preterm infants cared for using this oximeter.

\section{EXPLANATION}

We are grateful to Masimo Corporation for promptly identifying the explanation. Oximeters determine saturation by measuring the ratio of red to infrared waveform intensities

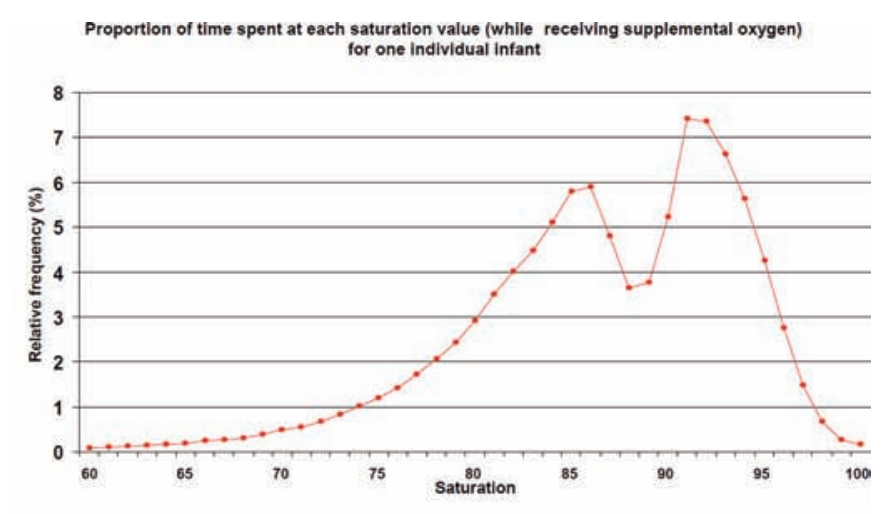

(a)

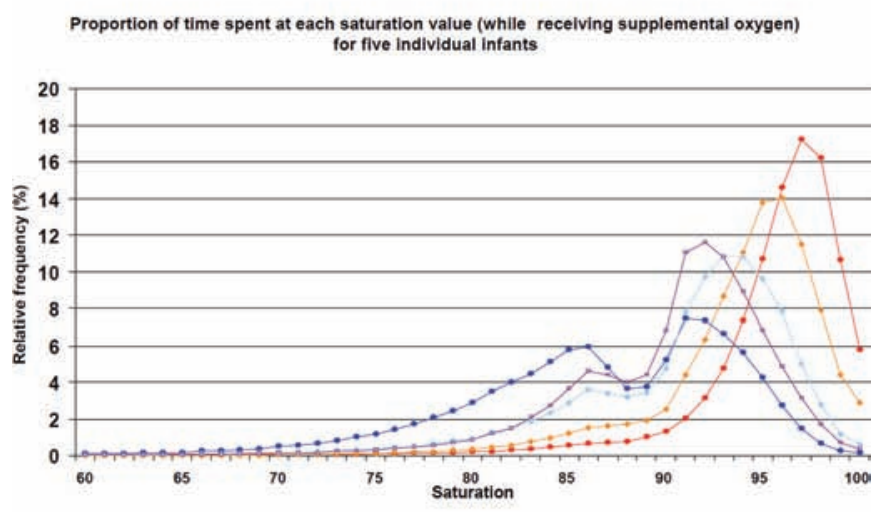

(b)

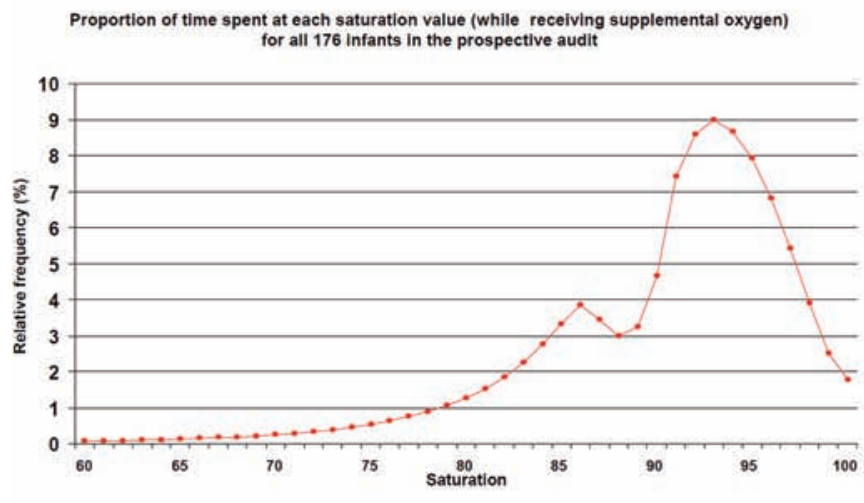

(c)

Figure 1 Frequency histograms for proportion of time spent at each saturation value while receiving supplemental oxygen in infants monitored using un-modified Masimo SET Radical pulse oximeters: (A) individual infant; (B) five individual infants; (C) cumulative data for all 176 infants in the prospective audit.

detected by the oximeter sensor (figure 2) and use a calibration curve to convert these ratios into saturation values. In development, this conversion is validated against arterial blood samples measured by a $\mathrm{CO}$-oximeter. The reduced frequency of $\mathrm{SpO}_{2}$ values of $87-90 \%$ is due to the calibration algorithm used by the oximeter. Inspection of the calibration curve in the area corresponding to $\mathrm{SpO}_{2}$ values of $87-90 \%$ shows a transition where the slope of the curve becomes steeper for a short section. The calibration curve has been generated by merging two separate curves and the change in slope is the 
Forward saturation equation

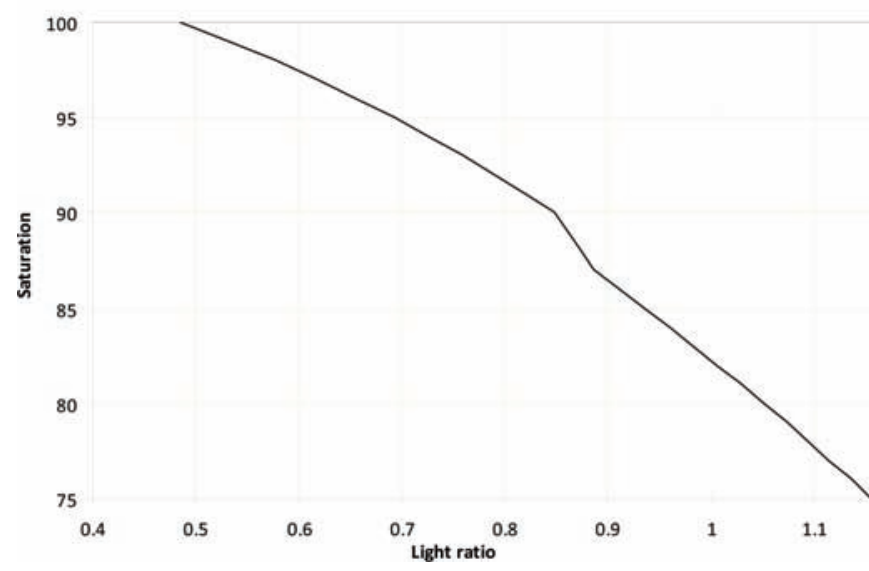

Figure 2 Plot of the ratio of red to infrared wavelength intensities and corresponding oxygen saturation (data supplied by Masimo Corporation).

move from one curve to the other. Where the slope is steeper, the $\mathrm{SpO}_{2}$ value returned increases more for each change in light ratio than would otherwise have been the case on either curve so that $\mathrm{SpO}_{2}$ readings of $87-90 \%$ become less frequent. From a $\mathrm{SpO}_{2}$ of $87 \%$ upwards, as the lower curve climbs to join the higher curve, $\mathrm{SpO}_{2}$ reads progressively a little higher than would be the case with the lower curve. This leads to an artefactual elevation of $\mathrm{SpO}_{2}$ readings that is maximal at a displayed value of $90 \%$ and is around $1.6 \%$ higher (effectively up to $2 \%$ because oximeters display whole numbers). This elevation gradually reduces in size as $\mathrm{SpO}_{2}$ increases further until it is no longer present at $\mathrm{SpO}_{2}$ values above $96 \%$.

The manufacturers indicate that the upper curve was used to provide saturation readings consistent with the tendency of manufacturers at the time that the oximeter was originally produced to adjust saturations in the 90 s upwards a little to obviate the effects of low levels of circulating variant haemoglobins. They added the lower curve, which is free of upward adjustment, in 2002 as newborn preterm infants were being targeted to lower saturations. The effect of this 'dual' curve is that the saturation values that are missing from the distribution in the region of $87-90 \%$ have been shifted upwards by up to $2 \%$ and there is a corresponding increase in the frequency of higher values.

Figure 3 shows $\mathrm{SpO}_{2}$ data gathered from an oxygen dependent preterm baby who was being cared for using a Nellcor Oximax N600x oximeter (Covidien-Nellcor, Boulder, Colorado, USA). The baby also had an unmodified Masimo SET Radical pulse oximeter attached, and the two simultaneous saturation profiles show the reduced number of values of $87-90 \%$ in the Masimo histogram and the higher number of $\mathrm{SpO}_{2}$ readings of 91-96\% that were obtained with the Masimo oximeter.

Masimo Corporation supplied new software with a revised conversion algorithm (board firmware V4.8 or higher) that relied on a single calibration curve and this was installed into all BOOST-II UK trial oximeters in December 2008. Figure 3B shows a paired comparison of saturation readings obtained from an oxygen dependent preterm infant cared for using an oximeter with the new saturation algorithm and simultaneously monitored with a standard Masimo SET Radical oximeter with the original algorithm.

Figure 4 shows paired comparisons of $\mathrm{SpO}_{2}$ values obtained from four infants whose clinical care was guided by one of four

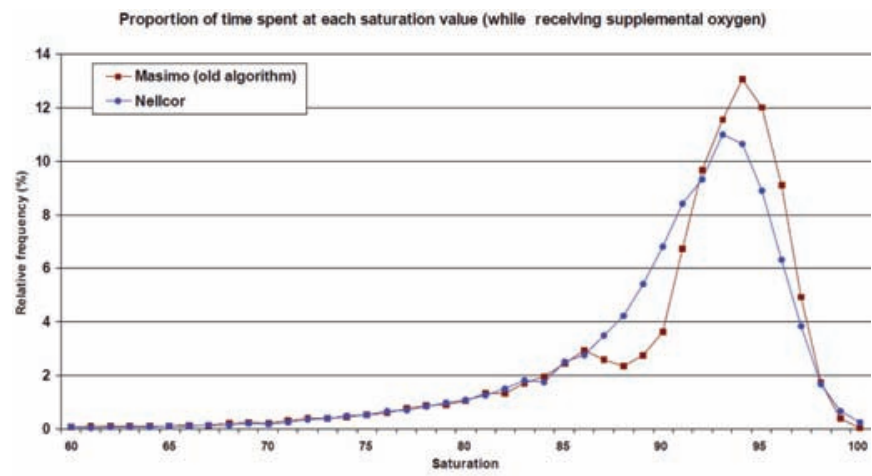

(a)

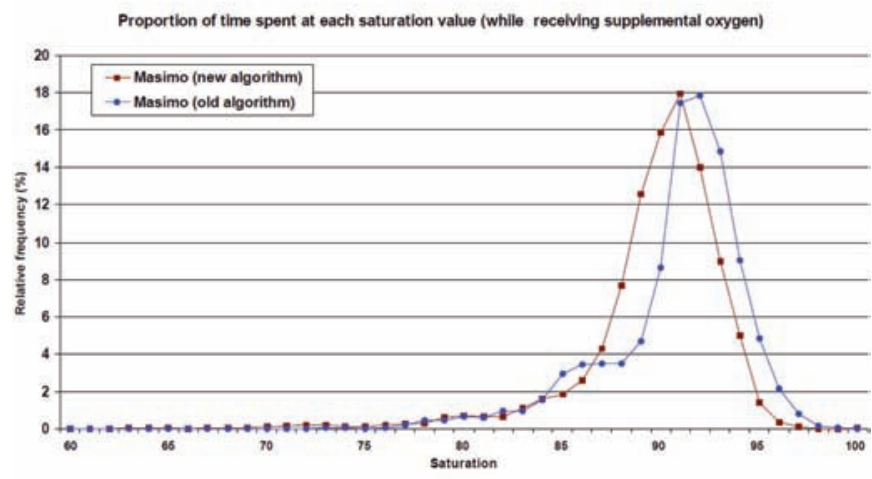

(b)

Figure 3 Frequency histograms for proportion of time spent at each saturation value while receiving supplemental oxygen: $(A)$ infant cared for using a Nellcor OxiMax N-600x oximeter with Nellcor OxiMax probe (Covidien-Nellcor, Boulder, Colorado, USA) and simultaneously monitored using a Masimo SET Radical oximeter with a Masimo LNOP NeoPt-L probe; (B) infant cared for using a Masimo SET Radical oximeter with the revised software algorithm and simultaneously monitored with a Masimo SET Radical oximeter with the original software algorithm.

different current commercially available oximeters and who also had $\mathrm{SpO}_{2}$ data gathered simultaneously with a Masimo SET Radical oximeter with the revised software. The revised Masimo software returned a distribution that was similar to that of the other four oximeters.

\section{DISCUSSION}

We have shown that when it is used to monitor preterm infants receiving supplemental oxygen, the Masimo SET Radical pulse oximeter returns a reduced number of values of $87-90 \%$. This is explained by the internal calibration algorithm, which changes in this region and adjusts $\mathrm{SpO}_{2}$ values above $87 \%$ upwards. A new software algorithm removed this artefact and returns a distribution of values that is similar to other current oximeters.

According to the product information, the oximeter is validated for accuracy in human blood studies on healthy adult volunteers in induced hypoxia studies in the range of $70-100 \%$ $\mathrm{SpO}_{2}$ against a laboratory $\mathrm{CO}$-oximeter. This variation equals $\pm 1 \mathrm{SD}$, which encompasses $68 \%$ of the population. The stated accuracy for neonates is $\pm 3 \%$, so by this standard at least $68 \%$ of the time the displayed $\mathrm{SpO}_{2}$ reading should be within $\pm 3 \%$ of the simultaneous $\mathrm{CO}$-oximeter value on an arterial blood sample. In this regard the original oximeter performs within all recommended accuracy standards. 
(a)

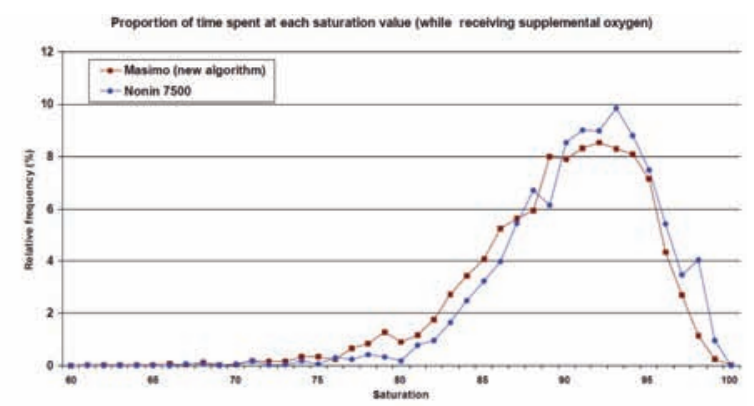

(b)

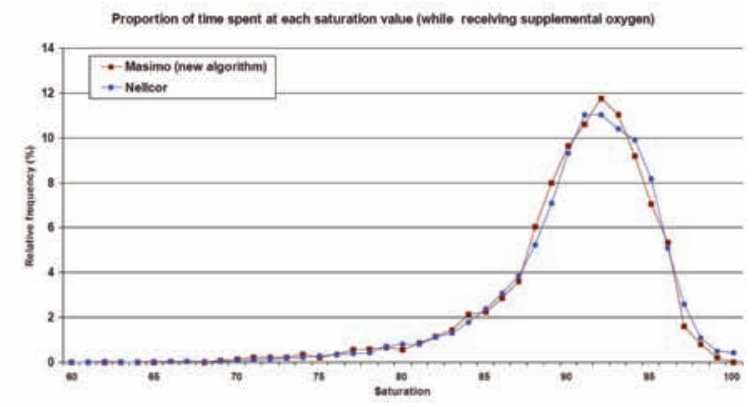

(c)

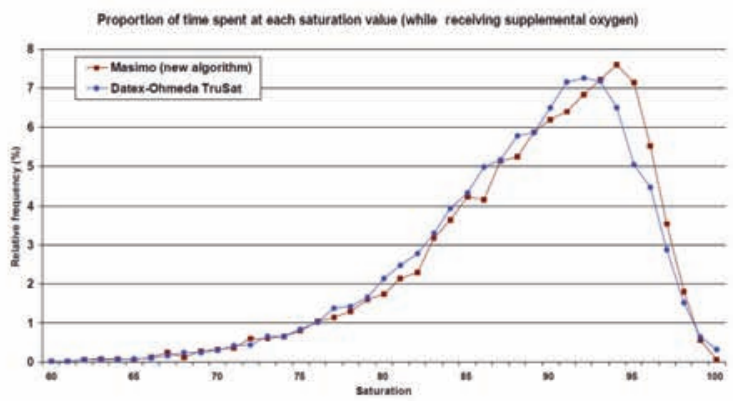

(d)

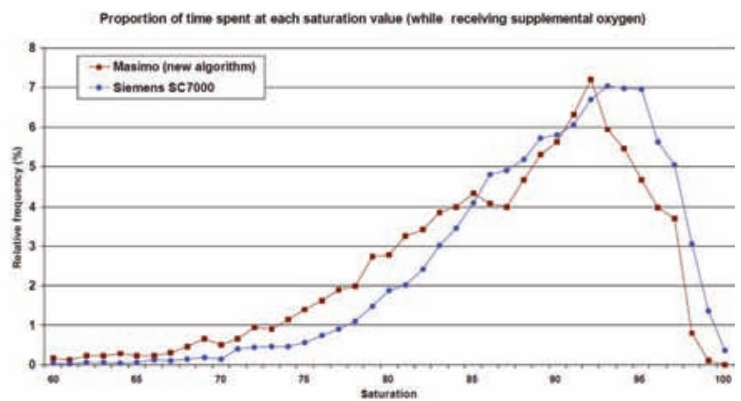

Figure 4 Frequency histograms for proportion of time spent at each saturation value while receiving supplemental oxygen for infants cared for using four commercially available oximeters and simultaneously monitored with a Masimo SET Radical oximeter with the revised software algorithm and a Masimo LNOP NeoPt-L probe: (A) Nonin 7500 using a 7000N Neonatal Flexiform II probe (Nonin Medical, Plymouth, Minnesota, USA); (B) oximeter with Nellcor OxiMax probe (Covidien-Nellcor, Boulder, Colorado, USA); (C) DatexOhmeda TruSat with an OxyTip sensitive skin probe (GE Healthcare, Chalfont St Giles, UK); (D) Siemens SC7000 (Siemens UK, Frimley, UK) with a Nellcor OxiMax probe.

Where $\mathrm{SpO}_{2}$ is targeted over a wide range, including values of $87 \%$ and $95 \%$, this artefact is not likely to be of clinical significance. However, oximeters are sometimes used to target a narrower range or to determine a threshold saturation. ${ }^{7-9}$ Under these circumstances the effect could be greater. Oximeters are used as a screening tool to identify borderline saturations in congenital heart disease. ${ }^{10} 11$ Some cases of duct dependent systemic circulation are not identified by this approach.
It is possible that a slight artefactual elevation of saturation between $87 \%$ and $95 \%$ could mask some cases. It is becoming usual for bronchopulmonary dysplasia to be categorised by whether or not a saturation of $90 \%$ is maintained breathing air. ${ }^{12-14} \mathrm{An}$ artefactual increase in $\mathrm{SpO}_{2}$ from $89 \%$ to $91 \%$ would result in fewer infants being categorised as having bronchopulmonary dysplasia and might influence the duration of supplemental oxygen exposure.

In the neonatal oxygen trials, infants have been targeted using modified Masimo Radical SET oximeters to maintain saturations either in the range $85-89 \%$ or $91-95 \% .{ }^{15}$ This artefact in the algorithm would be expected to reduce the number of saturation values in the target range in the group targeted to lower saturations. This might make this lower saturation target group harder to maintain within range, leading to more oxygen adjustments and greater variability in saturation, including time spent with high or low saturations.

When these observations were explained, Masimo Corporation provided the revised software, which eliminated the artefact, removing the deficit of values in the range $87-90 \%$, and reducing the uplift in saturations. With assistance from Masimo the new software algorithm was installed into the oximeters of the BOOST-II UK trial in December 2008 and into the Australian and Canadian oxygen trial oximeters in early 2009 , so that the final results of the international collaboration will be as generalisable as possible to current oximeters from Masimo and other manufacturers.

The SUPPORT trial was the first of the oxygen trials to complete recruitment. Outcomes to hospital discharge were published recently. ${ }^{9}$ There was a large and highly significant reduction in retinopathy of prematurity in the group who had their oxygen saturation targeted lower. The same group showed a slight excess of mortality, of borderline conventional statistical significance. The data monitoring committees of the ongoing trials in the UK, Australia and New Zealand and Canada reviewed their individual trial data in light of the SUPPORT trial results and independently recommended continuing recruitment to all three trials. The SUPPORT trial was completed before the oximeter modifications. Because the ongoing trials began evaluating an intervention that results in different saturation distributions, it is critically important to the generalisability of the trial results that a pooled analysis of the data should describe outcomes using either algorithm.

In conclusion, we have shown that the algorithm used by one generation of the Masimo SET Radical oximeter returns a distribution of saturation values above $87 \%$ that is different from other oximeters. This could be important if the device is being used to target saturation values in a narrow range or to evaluate threshold saturation values. This finding has important implications for the interpretation of emerging evidence in neonatal intensive care.

Acknowledgement The authors are grateful to the nursing staff and neonatologists of the units participating in the BOOST-II UK trial for contributing to the pretrial quality improvement exercise and enabling this important information to be identified and corrected. The authors thank Masimo Corporation for enabling the trials to take place and for promptly explaining the oximeter observations and providing the revised software.

Competing interests None.

Provenance and peer review Not commissioned; externally peer reviewed

\section{REFERENCES}

1. Collins MP, Lorenz JM, Jetton JR, et al. Hypocapnia and other ventilationrelated risk factors for cerebral palsy in low birth weight infants. Pediatr Res 2001; 50:712-19. 
2. Tin W, Milligan DW, Pennefather $\mathrm{P}$, et al. Pulse oximetry, severe retinopathy, and outcome at one year in babies of less than 28 weeks gestation. Arch Dis Child Fetal Neonatal Ed 2001;84:F106-10.

3. Askie LM, Henderson-Smart DJ, Irwig L, et al. Oxygen-saturation targets and outcomes in extremely preterm infants. N Engl J Med 2003;349:959-67.

4. Chow LC, Wright KW, Sola A. Can changes in clinical practice decrease the incidence of severe retinopathy of prematurity in very low birth weight infants? Pediatrics 2003;111:339-45.

5. McColm JR, Cunningham S, Wade J, et al. Hypoxic oxygen fluctuations produce less severe retinopathy than hyperoxic fluctuations in a rat model of retinopathy of prematurity. Pediatr Res 2004;55:107-13.

6. Deulofeut R, Critz A, Adams-Chapman I, et al. Avoiding hyperoxia in infants $<$ or $=1250 \mathrm{~g}$ is associated with improved short- and long-term outcomes. J Perinatol 2006;26:700-5.

7. Hagadorn JI, Furey AM, Nghiem TH, et al. Achieved versus intended pulse oximeter saturation in infants born less than 28 weeks' gestation: the AVIOx study. Pediatrics 2006;118:1574-82.

8. Claure N, D'Ugard C, Bancalari E. Automated adjustment of inspired oxygen in preterm infants with frequent fluctuations in oxygenation: a pilot clinical trial. J Pediatr 2009;155:640-5.e1-2.
9. SUPPORT Study Group of the Eunice Kennedy Shriver NICHD Neonatal Research Network. Target ranges of oxygen saturation in extremely preterm infants. N Engl J Med 2010;362:1959-69.

10. de-Wahl Granelli A, Wennergren M, Sandberg K, et al. Impact of pulse oximetry screening on the detection of duct dependent congenital heart disease: a Swedish prospective screening study in 39,821 newborns. BMJ 2009;338:a3037.

11. Meberg A, Brügmann-Pieper S, Due R Jr, et al. First day of life pulse oximetry screening to detect congenital heart defects. J Pediatr 2008;152:761-5.

12. Walsh MC, Wilson-Costello D, Zadell A, et al. Safety, reliability, and validity of a physiologic definition of bronchopulmonary dysplasia. J Perinatol 2003;23:451-6.

13. Walsh MC, Yao 0, Gettner P, et al. Impact of a physiologic definition on bronchopulmonary dysplasia rates. Pediatrics 2004;114:1305-11.

14. Ballard RA, Truog WE, Cnaan A, et al. Inhaled nitric oxide in preterm infants undergoing mechanical ventilation. N Engl J Med 2006;355:343-53.

15. Askie LM, Simes J, Tarnow-Mordi W, et al. NeOProM Collaboration: a Prospective, Individual Patient Data Meta-Analysis Assessing Appropriate Levels of Oxygen Saturation for Extremely Preterm Infants: University of Sydney. http:// sydney.edu.au/medicine/public-health/research/projects.php (accessed 1 January 2010). 